\title{
AN OPTIMAL ALGORITHM FOR THE MAXIMUM-DENSITY SEGMENT PROBLEM*
}

\author{
KAI-MIN CHUNG ${ }^{\dagger}$ AND HSUEH-I LU
}

\begin{abstract}
We address a fundamental problem arising from analysis of biomolecular sequences. The input consists of two numbers $w_{\min }$ and $w_{\max }$ and a sequence $S$ of $n$ number pairs $\left(a_{i}, w_{i}\right)$ with $w_{i}>0$. Let segment $S(i, j)$ of $S$ be the consecutive subsequence of $S$ between indices $i$ and $j$. The density of $S(i, j)$ is $d(i, j)=\left(a_{i}+a_{i+1}+\cdots+a_{j}\right) /\left(w_{i}+w_{i+1}+\cdots+w_{j}\right)$. The maximum-density segment problem is to find a maximum-density segment over all segments $S(i, j)$ with $w_{\min } \leq w_{i}+w_{i+1}+\cdots+w_{j} \leq w_{\max }$. The best previously known algorithm for the problem, due to Goldwasser, Kao, and Lu [Proceedings of the Second International Workshop on Algorithms in Bioinformatics, R. Guigó and D. Gusfield, eds., Lecture Notes in Comput. Sci. 2452, SpringerVerlag, New York, 2002, pp. 157-171], runs in $O\left(n \log \left(w_{\max }-w_{\min }+1\right)\right)$ time. In the present paper, we solve the problem in $O(n)$ time. Our approach bypasses the complicated right-skew decomposition, introduced by Lin, Jiang, and Chao [J. Comput. System Sci., 65 (2002), pp. 570-586]. As a result, our algorithm has the capability to process the input sequence in an online manner, which is an important feature for dealing with genome-scale sequences. Moreover, for a type of input sequences $S$ representable in $O(m)$ space, we show how to exploit the sparsity of $S$ and solve the maximumdensity segment problem for $S$ in $O(m)$ time.
\end{abstract}

Key words. bioinformatics, biological sequence analysis, maximum-density segment, slope selection, computational geometry, sequence algorithm, data structure

AMS subject classifications. 68P05, 68Q25, 68R05, 68U05, 68W05, 68W40, 92-08, 92D20

DOI. $10.1137 /$ S0097539704440430

1. Introduction. We address the following fundamental problem: The input consists of two numbers $w_{\min }$ and $w_{\max }$ and a sequence $S$ of number pairs $\left(a_{i}, w_{i}\right)$ with $w_{i}>0$ for $i=1, \ldots, n$. A segment $S(i, j)$ is a consecutive subsequence of $S$ from position $i$ to position $j$. The width $w(i, j)$ of $S(i, j)$ is $w_{i}+w_{i+1}+\cdots+w_{j}$. The density $d(i, j)$ of $S(i, j)$ is $\left(a_{i}+a_{i+1}+\cdots+a_{j}\right) / w(i, j)$. It is not difficult to see that with an $O(n)$-time preprocessing to compute all $O(n)$ prefix sums $a_{1}+a_{2}+\cdots+a_{j}$ and $w_{1}+w_{2}+\cdots+w_{j}$, the density of any segment can be computed in $O(1)$ time. $S(i, j)$ is feasible if $w_{\min } \leq w(i, j) \leq w_{\max }$. The maximum-density segment problem is to find a maximum-density segment over all $O\left(n\left(w_{\max }-w_{\min }+1\right)\right)$ feasible segments.

This problem arises from the investigation of the nonuniformity of nucleotide composition within genomic sequences, which was first revealed through thermal melting and gradient centrifugation experiments $[21,28]$. The GC content of the DNA sequences in all organisms varies from $25 \%$ to $75 \%$. GC-ratios have the greatest variation among bacterial DNA sequences, while the typical GC-ratios of mammalian

${ }^{*}$ Received by the editors February 3, 2004; accepted for publication (in revised form) July 7, 2004; published electronically December 1, 2004. A preliminary version of this paper appeared in Proceedings of the 11th Annual European Symposium on Algorithms (Budapest, Hungary, 2003), G. Di Battista and U. Zwick, eds., Lecture Notes in Comput. Sci. 2832, Springer-Verlag, New York, 2003, pp. 136-147.

http://www.siam.org/journals/sicomp/34-2/44043.html

$\dagger$ Institute of Information Science, Academia Sinica. Part of this work was done while this author was an undergraduate student in the Department of Computer Science and Information Engineering, National Taiwan University.

${ }^{\ddagger}$ Corresponding author. Institute of Information Science, Academia Sinica, 128 Academia Road, Section 2, Taipei 115, Taiwan, Republic of China (hil@iis.sinica.edu.tw, www.iis.sinica.edu.tw/ hil/). This author's research was supported in part by NSC grants 91-2215-E-001-001 and 92-2218-E-001001. 
genomes stay in the range $45-50 \%$. Despite intensive research effort in the past two decades, the underlying causes of the observed heterogeneity remain debatable $[2,3,5,8,9,10,11,18,40,42]$. Researchers [32,39] observed that the extent of the compositional heterogeneity in a genomic sequence strongly correlates with its GC content regardless of genome size. Other investigations showed that gene length [7], gene density [44], patterns of codon usage [37], distribution of different classes of repetitive elements [7,38], number of isochores [2], lengths of isochores [32], and recombination rate within chromosomes [12] are all correlated with GC content. More research related to GC-rich segments can be found in $[16,17,20,23,29,31,35,41,43]$ and references therein.

In the most basic form of the maximum-density segment problem, the sequence $S$ corresponds to the given DNA sequence, where $a_{i}=1$ if the corresponding nucleotide in the DNA sequence is a $\mathrm{G}$ or $\mathrm{C}$, and $a_{i}=0$ otherwise. In the work of Huang [19], sequence entries took on values of $p$ and $1-p$ for some real number $0 \leq p \leq 1$. More generally, we can look for regions where a given set of patterns occurs very often. In such applications, $a_{i}$ could be the relative frequency with which the corresponding DNA segments appear in the given patterns. Further natural applications of this problem can be designed for sophisticated sequence analysis such as mismatch density [36], ungapped local alignments [1], annotated multiple sequence alignments [39], promoter mapping [22], and promoter recognition [33].

For the uniform case, i.e., $w_{i}=1$ for all indices $i$, Nekrutenko and Li [32] and Rice, Longden, and Bleasby [34] employed algorithms for the case $w_{\min }=w_{\max }$, which is trivially solvable in $O(n)$ time. More generally, when $w_{\min } \neq w_{\max }$, the problem is also easily solvable in $O\left(n\left(w_{\max }-w_{\min }+1\right)\right)$ time, linear in the number of feasible segments. Huang [19] studied the case where $w_{\max }=n$, i.e., there is effectively no upper bound on the width of the desired maximum-density segments. He observed that an optimal segment exists with width at most $2 w_{\min }-1$. Therefore, this case is equivalent to the case with $w_{\max }=2 w_{\min }-1$ and can be solved in $O\left(n w_{\min }\right)$ time in a straightforward manner. Lin, Jiang, and Chao [27] gave an $O\left(n \log w_{\min }\right)$-time algorithm for this case based on right-skew decompositions of a sequence. (See [26] for related software.) The case with general $w_{\max }$ was first investigated by Goldwasser, Kao, and $\mathrm{Lu}[13,14]$, who gave an $O(n)$-time algorithm for the uniform case. Recently, Kim [25] showed an alternative algorithm based upon a geometric interpretation of the problem, which basically relates the maximum-density segment problem to the fundamental slope selection problem in computational geometry $[4,6,24,30]$. Unfortunately, Kim's analysis of time complexity has a flaw which seems difficult to fix. ${ }^{1}$ For the general (i.e., nonuniform) case, Goldwasser, Kao, and Lu [13] also gave an $O\left(n \log \left(w_{\max }-w_{\min }+1\right)\right)$-time algorithm. By bypassing the complicated preprocessing step required in [13], we successfully reduce the time required for the general case down to $O(n)$. Our result is based upon the following set of equations, stating that the order of $d(x, y), d(y+1, z)$, and $d(x, z)$ with $x \leq y<z$ can be determined by the

\footnotetext{
${ }^{1} \mathrm{Kim}$ claims that all the progressive updates of the lower convex hulls $L_{j} \cup R_{j}$ can be done in overall linear time. The paper only sketches how to obtain $L_{j+1} \cup R_{j+1}$ from $L_{j} \cup R_{j}$. (See the fourth-to-last paragraph of p. 340 in [25].) Unfortunately, Kim seems to overlook the marginal cases when the upper bound $w_{\max }$ forces the $p_{z}$ of $L_{j} \cup R_{j}$ to be deleted from $L_{j+1} \cup R_{j+1}$. As a result, obtaining $L_{j+1} \cup R_{j+1}$ from $L_{j} \cup R_{j}$ could be much more complicated than Kim's sketch. A naive implementation of Kim's algorithm still takes $\Omega\left(n\left(w_{\max }-w_{\min }+1\right)\right)$ time in the worst case. We believe that any correct implementation of Kim's algorithm requires $\Omega\left(n \log \left(w_{\max }-w_{\min }+1\right)\right)$ time in the worse case.
} 

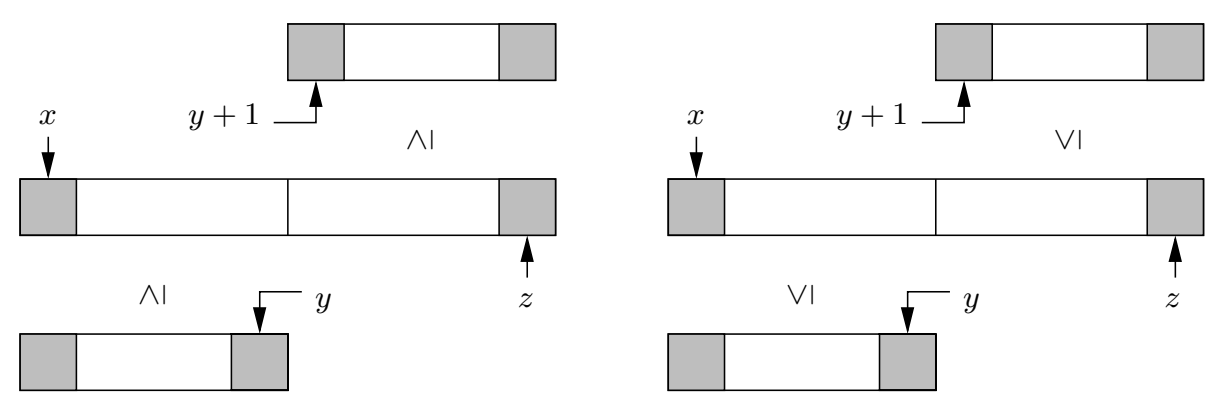

FIG. 1.1. An illustration for (1.1): There exist only two possibilities for the order among $d(x, y), d(x, z), d(y+1, z)$.

order of any two of them:

$$
\begin{aligned}
& d(x, y) \leq d(y+1, z) \Leftrightarrow d(x, y) \leq d(x, z) \Leftrightarrow d(x, z) \leq d(y+1, z), \\
& d(x, y) \geq d(y+1, z) \Leftrightarrow d(x, y) \geq d(x, z) \Leftrightarrow d(x, z) \geq d(y+1, z) .
\end{aligned}
$$

(Both equations can be easily verified by observing the existence of some number $\rho$ with $0<\rho<1$ and $d(x, z)=\rho \cdot d(x, y)+(1-\rho) \cdot d(y+1, z)$. See Figure 1.1.) Our algorithm is capable of processing the input sequence in an online manner, which is an important feature for dealing with genome-scale sequences.

For bioinformatics applications, e.g., in $[1,22,33,36,39]$, the input sequence $S$ is usually very sparse. That is, $S$ can be represented by $m=o(n)$ triples

$$
\left(a_{1}^{\prime}, w_{1}^{\prime}, n_{1}\right),\left(a_{2}^{\prime}, w_{2}^{\prime}, n_{2}\right), \ldots,\left(a_{m}^{\prime}, w_{m}^{\prime}, n_{m}\right)
$$

with $0=n_{0}<n_{1}<n_{2}<\cdots<n_{m}=n$ to signify that $\left(a_{i}, w_{i}\right)=\left(a_{j}^{\prime}, w_{j}^{\prime}\right)$ holds for all indices $i$ and $j$ with $n_{j-1}<i \leq n_{j}$ and $1 \leq j \leq m$. If $w_{j}^{\prime}=1$ holds for all $1 \leq j \leq m$, we show how to exploit the sparsity of $S$ and solve the maximum-density problem for $S$ given in the above compact representation in $O(m)$ time.

The remainder of the paper is organized as follows. Section 2 shows the main algorithm. Section 3 explains how to cope with the simple case that the width upper bound $w_{\max }$ is ineffective. Section 4 takes care of the more complicated case that $w_{\max }$ is effective. Section 5 explains how to exploit the sparsity of the input sequence for the uniform case.

2. The main algorithm. For any integers $x$ and $y$, let $[x, y]$ denote the set $\{x, x+1, \ldots, y\}$. Without loss of generality, we may assume that $w_{1}+w_{2}+\cdots+w_{n} \geq$ $w_{\min }$ and $w_{i} \leq w_{\max }$ holds for each $i=1,2, \ldots, n$. Throughout the paper, we need the following definitions and notation with respect to the input length- $n$ sequence $S$ and width bounds $w_{\min }$ and $w_{\max }$. Define $\phi(x, y)$ to be the largest index $z \in[x, y]$ that minimizes $d(x, z)$. That is, $S(x, \phi(x, y))$ is the longest minimum-density prefix of $S(x, y)$. Let $j_{0}$ be the smallest index with $w\left(1, j_{0}\right) \geq w_{\min }$. Let $J=\left[j_{0}, n\right]$. For each $j \in J$, let $\ell_{j}$ be the smallest index $i$ with $w(i, j) \leq w_{\max }$, and let $r_{j}$ be the largest index $i$ with $w(i, j) \geq w_{\min }$. That is, $S(i, j)$ is feasible if and only if $i \in\left[\ell_{j}, r_{j}\right]$. (Figure 2.1 is an illustration for the definitions of $\ell_{j}$ and $r_{j}$.) Clearly, for the uniform case, we have $\ell_{i+1}=\ell_{i}+1$ and $r_{i+1}=r_{i}+1$. As for the general case, we know only that $\ell_{j}$ and $r_{j}$ are both (not necessarily strictly) increasing. One can easily compute all $\ell_{j}$ and $r_{j}$ in $O(n)$ time.

Let $i_{j}^{*}$ be the largest index $k \in\left[\ell_{j}, r_{j}\right]$ with $d(k, j)=\max \left\{d(i, j) \mid i \in\left[\ell_{j}, r_{j}\right]\right\}$. There must be an index $j^{*}$ such that $S\left(i_{j^{*}}^{*}, j^{*}\right)$ is a maximum-density segment of 


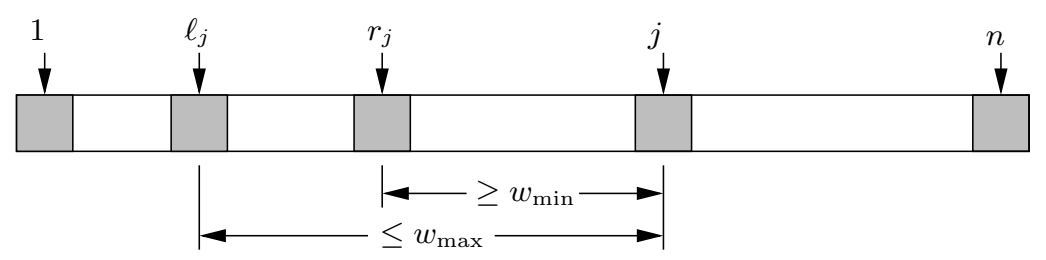

FIG. 2.1. An illustration for the definitions of $\ell_{j}$ and $r_{j}$.

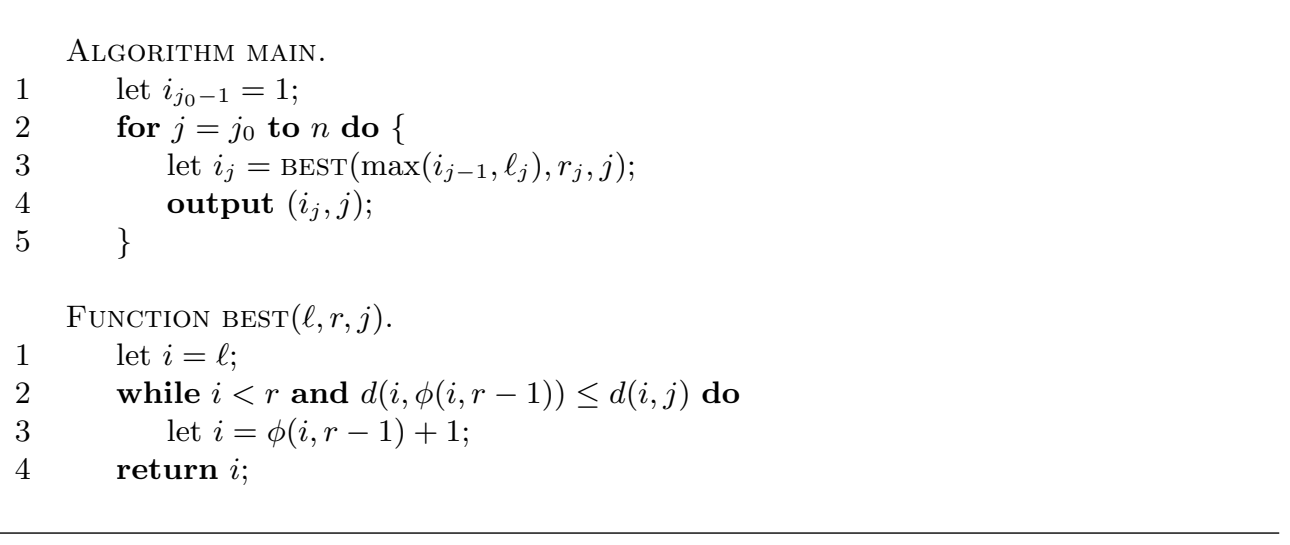

FIG. 2.2. Our main algorithm.

$S$. Therefore, a natural but seemingly difficult possibility for solving the maximumdensity segment problem would be to compute $i_{j}^{*}$ for all indices $j \in J$ in $O(n)$ time. Instead, our approach is to compute a pair $\left(i_{j}, j\right)$ of indices with $i_{j} \in\left[\ell_{j}, r_{j}\right]$ for each index $j \in J$ by the algorithm shown in Figure 2.2. More specifically, each iteration of our algorithm, based upon (1.1), keeps chopping off the lowest-density prefix without affecting the feasibility of the remaining segment until its density does not go any higher. For brevity of presentation, each algorithm throughout the paper outputs a linear number of index pairs $(i, j)$. (See, e.g., step 4 of algorithm MAIN shown in Figure 2.2.) Clearly, it takes a linear-time postprocessing for the produced index pairs to obtain one that maximizes $d(i, j)$. The rest of the section ensures the correctness of our algorithm by showing $i_{j^{*}}=i_{j^{*}}^{*}$, and thus reduces the maximum-density segment problem to implementing our algorithm to run in $O(n)$ time.

Lemma 2.1. The index returned by function call $\operatorname{BEST}(\ell, r, j)$ is the largest index $i \in[\ell, r]$ that maximizes $d(i, j)$.

Proof. Let $i^{*}$ be the largest index in $[\ell, r]$ that maximizes $d(i, j)$, i.e., $d\left(i^{*}, j\right)=$ $\max _{i \in[\ell, r]} d(i, j)$. Let $i_{j}$ be the index returned by function call $\operatorname{BEST}(\ell, r, j)$. We show $i_{j}=i^{*}$ as follows. If $i_{j}<i^{*}$, then $i_{j}<r$. By the condition of the while-loop at step 2 of BEST, we know $d\left(i_{j}, \phi\left(i_{j}, r-1\right)\right)>d\left(i_{j}, j\right)$. By $d\left(i_{j}, j\right) \leq d\left(i^{*}, j\right)$ and $(1.1)$, we have $d\left(i_{j}, i^{*}-1\right) \leq d\left(i_{j}, j\right)$. It follows that $d\left(i_{j}, i^{*}-1\right)<d\left(i_{j}, \phi\left(i_{j}, r-1\right)\right)$, contradicting the definition of $\phi\left(i_{j}, r-1\right)$.

On the other hand, suppose that $i_{j}>i^{*}$. By definition of BEST, there must be an index $i \in[\ell, r]$ with $i<r, d(i, \phi(i, r-1)) \leq d(i, j)$, and $i \leq i^{*}<\phi(i, r-1)+1$. If $i=i^{*}$, by $(1.1)$ we have $d\left(i^{*}, \phi\left(i^{*}, r-1\right)\right) \leq d\left(i^{*}, j\right) \leq d\left(\phi\left(i^{*}, r-1\right)+1, j\right)$, where the last inequality contradicts the definition of $i^{*}$. Now that $i<i^{*}$, we have $d\left(i^{*}, j\right) \geq$ $d(i, j) \geq d\left(i, i^{*}-1\right) \geq d(i, \phi(i, r-1)) \geq d\left(i^{*}, \phi(i, r-1)\right)$, where (a) the first inequality is 
by definition of $i^{*}$, (b) the second inequality is by (1.1) and the first inequality, (c) the third inequality is by $i^{*} \leq \phi(i, r-1)$ and the definition of $\phi(i, r-1)$, and (d) the last inequality is by (1.1) and the third inequality. It follows from $d\left(i^{*}, j\right) \geq d\left(i^{*}, \phi(i, r-1)\right)$ and (1.1) that $d(\phi(i, r-1)+1, j) \geq d\left(i^{*}, j\right)$, contradicting the definition of $i^{*}$ by $i^{*}<\phi(i, r-1)+1$.

THEOREM 2.2. Algorithm MAIN correctly solves the maximum-density segment problem.

Proof. We prove the theorem by showing $i_{j^{*}}=i_{j^{*}}^{*}$. By $\ell_{j_{0}}=i_{j_{0}-1}=1$ and Lemma 2.1, the equality holds if $j^{*}=j_{0}$. The rest of the proof assumes $j^{*}>j_{0}$. By Lemma 2.1 and $\ell_{j^{*}} \leq i_{j^{*}}^{*}$, it suffices to ensure $i_{j^{*}-1} \leq i_{j^{*}}^{*}$. Assume for contradiction that there is an index $j \in\left[j_{0}, j^{*}-1\right]$ with $i_{j-1} \leq i_{j^{*}}^{*}<i_{j}$. By $j<j^{*}$, we know $\ell_{j} \leq i_{j^{*}}^{*}$. By Lemma 2.1 and $\max \left(\ell_{j}, i_{j-1}\right) \leq i_{j^{*}}^{*}<i_{j} \leq r_{j}$, we have $d\left(i_{j}, j\right) \geq d\left(i_{j^{*}}^{*}, j\right)$. It follows from (1.1) and $i_{j^{*}}^{*}<i_{j}$ that $d\left(i_{j^{*}}^{*}, j\right) \geq d\left(i_{j^{*}}^{*}, i_{j}-1\right)$. By $\ell_{j^{*}} \leq i_{j^{*}}^{*}<i_{j} \leq r_{j^{*}}$ and the definition of $j^{*}$, we know $d\left(i_{j^{*}}^{*}, j^{*}\right)>d\left(i_{j}, j^{*}\right)$. It follows from $i_{j^{*}}^{*}<i_{j}$ and (1.1) that $d\left(i_{j^{*}}^{*}, i_{j}-1\right)>d\left(i_{j^{*}}^{*}, j^{*}\right)$. Therefore, $d\left(i_{j}, j\right) \geq d\left(i_{j^{*}}^{*}, j\right) \geq d\left(i_{j^{*}}^{*}, i_{j}-1\right)>$ $d\left(i_{j^{*}}^{*}, j^{*}\right)$, contradicting the definition of $j^{*}$.

One can verify that the value of $i$ increases by at least 1 each time step 3 of BEST is executed. Therefore, to implement the algorithm to run in $O(n)$ time, it suffices to maintain a data structure to support an $O(1)$-time query for each $\phi\left(i, r_{j}-1\right)$ in step 2 of BEST.

3. Coping with ineffective width upper bound. When $w_{\max }$ is ineffective, i.e., $w_{\max } \geq w(1, n)$, we have $\ell_{j}=1$ for all $j \in J$. Therefore, the function call in step 3 of MAIN is exactly $\operatorname{BEST}\left(i_{j-1}, r_{j}, j\right)$. Moreover, during the execution of the function call $\operatorname{BEST}\left(i_{j-1}, r_{j}, j\right)$, the value of $i$ can only be $i_{j-1}, \phi\left(i_{j-1}, r_{j}-1\right)+1$, $\phi\left(\phi\left(i_{j-1}, r_{j}-1\right)+1, r_{j}-1\right)+1, \ldots, r_{j}$. Suppose that a subroutine call to UPDATE $(j)$ yields an array $\Phi$ of indices and two indices $p$ and $q$ of $\Phi$ with $p \leq q$ and $\Phi[p]=i_{j-1}$ such that the following condition holds.

Condition $C_{j}: \Phi[q]=r_{j}$ and $\Phi[t]=\phi\left(\Phi[t-1], r_{j}-1\right)+1$ holds for each index $t \in[p+1, q]$. (See Figure 3.1 for an illustration.)

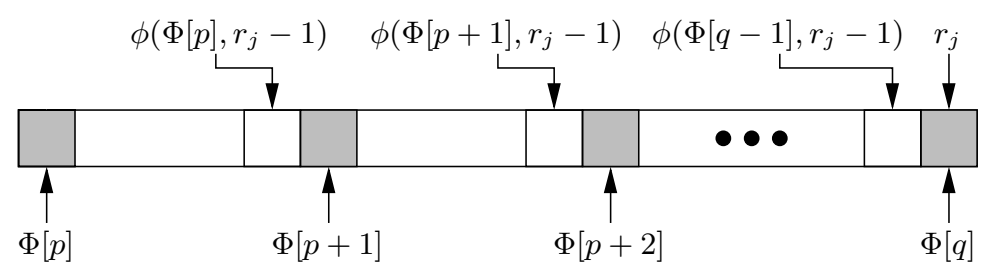

FIG. 3.1. An illustration for Condition $C_{j}$.

Then, the subroutine call to $\operatorname{BEST}\left(i_{j-1}, r_{j}, j\right)$ can clearly be replaced by $\operatorname{LBEST}(j)$, as defined in Figure 3.2. That is, $\operatorname{LBEST}(j)$ can access the value of each $\phi\left(i, r_{j}-1\right)$ by looking up $\Phi$ in $O(1)$ time. It remains to show how to implement $\operatorname{UPDATE}(j)$ such that all $O(n)$ subroutine calls to UPDATE from step 3 of LMAIN run in overall $O(n)$ time. With the initialization of letting $p=q=r_{j_{0}-1}=\Phi[1]=1$, as described at step 1 of algorithm LMAIN, Condition $C_{j_{0}-1}$ clearly holds before the subroutine call to $\operatorname{UPDATE}\left(j_{0}\right)$. The following lemma is crucial in ensuring the correctness and efficiency of our implementation shown in Figure 3.2.

LEMma 3.1. For each index $j \in J$, the following statements hold:

1. If Condition $C_{j-1}$ holds right before calling UPDATE $(j)$, then Condition $C_{j}$ holds right after the subroutine call. 


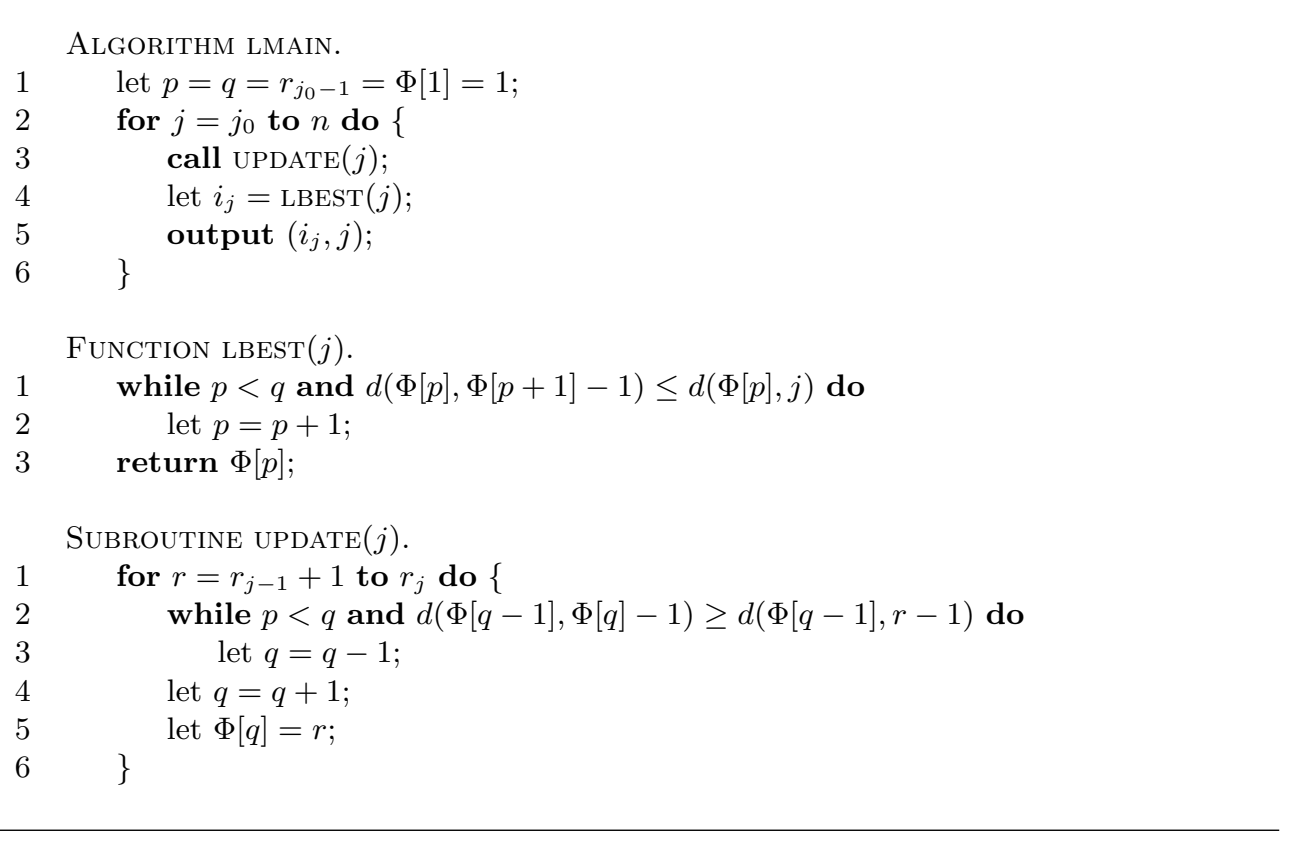

FIG. 3.2. An efficient implementation for the case that $w_{\max }$ is ineffective.

2. If Condition $C_{j}$ holds right before calling $\operatorname{LBEST}(j)$, then the index returned by the function call is exactly that returned by $\operatorname{BEST}(\Phi[p], \Phi[q], j)$.

Proof. Statement 1. We need the following condition.

Condition $D_{r}: \Phi[q]=r$ and $\Phi[t]=\phi(\Phi[t-1], r-1)+1$ holds for each index $t \in[p+1, q]$.

Clearly, Condition $C_{j}$ is exactly Condition $D_{r_{j}}$. To prove the statement, we show that if Condition $D_{r-1}$ holds, then Condition $D_{r}$ holds right after executing steps $2-5$ of UPDATE (i.e., an iteration of the for-loop of UPDATE), although the value of $q$ may change.

Consider the moment when step 4 of UPDATE is about to be executed. We first show that if $p<q$, then $\Phi[t]=\phi(\Phi[t-1], r-1)+1$ holds for each $t \in[p+1, q]$. By the definition of $\phi$, we have

$$
\phi(\ell, r-1)= \begin{cases}r-1 & \text { if } d(\ell, \phi(\ell, r-2)) \geq d(\ell, r-1), \\ \phi(\ell, r-2) & \text { otherwise. }\end{cases}
$$

By Condition $D_{r-1}$, we know $\phi(\Phi[q-1], r-2)=\Phi[q]-1$. With $\ell=\Phi[q-1]$ plugged into (3.1), we know that if $d(\Phi[q-1], \Phi[q]-1)<d(\Phi[q-1], r-1)$, then $\phi(\Phi[q-1], r-1)=\phi(\Phi[q-1], r-2)$. It follows from the condition of step 2 of UPDATE that $\phi(\Phi[q-1], r-1)=\phi(\Phi[q-1], r-2)$. Furthermore, if $\phi(\ell, r-2)<r-2$, then one can prove as follows that $\phi(\phi(\ell, r-2)+1, r-1)=\phi(\phi(\ell, r-2)+1, r-2)$ implies $\phi(\ell, r-1)=\phi(\ell, r-2)$.

Let $m=\phi(\ell, r-2)$. By $\phi(m+1, r-1)=\phi(m+1, r-2)$, we have $d(m+1, \phi(m+1, r-1))<d(m+1, r-1)$. By definition of $\phi$ and (1.1), we have $d(\ell, m)<d(\ell, \phi(m+1, r-1))<d(m+1, \phi(m+1, r-1))$. As a result, we have $d(\ell, m)<d(m+1, r-1)$, which by (1.1) implies $d(\ell, m)<d(\ell, r-1)$. Thus $\phi(\ell, r-1)=\phi(\ell, r-2)$. 
Therefore, $\phi(\Phi[t], r-1)=\phi(\Phi[t], r-2)$ implies $\phi(\Phi[t-1], r-1)=\phi(\Phi[t-1], r-2)$. As a result, $\phi(\Phi[t], r-1)=\phi(\Phi[t], r-2)$ holds for each $t \in[p, q-1]$. By Condition $D_{r-1}$, we know $\Phi[t]=\phi(\Phi[t-1], r-1)+1$ holds for each $t \in[p+1, q]$.

Since the value of $q$ will be incremented by 1 after executing step 4 of UPDATE and $\Phi[q+1]$ will equal $r$ after executing step 5 of UPDATE, it remains to show $\phi(\Phi[q], r-1)=$ $r-1$. Clearly, the equality holds if $\Phi[q]=r-1$, i.e., step 3 was not executed. If step 3 was executed at least once, then we know $d(\Phi[q], \Phi[q+1]-1) \geq d(\Phi[q], r-1)$, i.e., $d(\Phi[q], \phi(\Phi[q], r-2)) \geq d(\Phi[q], r-1)$. By plugging $\ell=\Phi[q]$ into (3.1), we know $\phi(\Phi[q], r-1)=r-1$.

Statement 2. By Condition $C_{j}$, one can easily verify that $\operatorname{LBEST}(j)$ is a faithful implementation of $\operatorname{BEST}(\Phi[p], \Phi[q], j)$. Therefore, the statement holds.

LEMMA 3.2. The implementation LMAIN solves the maximum-density problem for the case with ineffective $w_{\max }$ in $O(n)$ time.

Proof. By Lemma 3.1(1) and the definitions of UPDATE and LBEST, both Condition $C_{j}$ and $\Phi[p]=i_{j-1}$ hold right after the subroutine call to UPDATE $(j)$. By Condition $C_{j}$ and Lemma 3.1(2), $\operatorname{LBEST}(j)$ is a faithful implementation of $\operatorname{BEST}(\Phi[p], \Phi[q], j)$. Therefore, the correctness of LMAIN follows from $\Phi[p]=i_{j-1}, \Phi[q]=r_{j}$, and Theorem 2.2 .

As for the efficiency of LMAIN, observe that $q-p \geq 0$ holds throughout the execution of LMAIN. Note that each iteration of the while-loops of LBEST and UPDATE decreases the value of $q-p$ by one. Since step 4 of UPDATE, which is the only place that increases the value of $q-p$, increases the value of $q-p$ by one for $O(n)$ times, the overall running time of LMAIN is $O(n)$.

4. Coping with effective width upper bound. In contrast to the previous simple case, when $w_{\max }$ is arbitrary, $\ell_{j}$ may not always be 1 . Therefore, the first argument of the function call in step 3 of MAIN could be $\ell_{j}$ with $\ell_{j}>i_{j-1}$. It seems quite difficult to update the corresponding data structure $\Phi$ in overall linear time such that both $\Phi[p]=\max \left(i_{j-1}, \ell_{j}\right)$ and Condition $C_{j}$ hold throughout the execution of our algorithm. To overcome this difficulty, our algorithm sticks with Condition $C_{j}$ but allows $\Phi[p]>\max \left(i_{j-1}, \ell_{j}\right)$. As a result, $\max _{j \in J} d\left(i_{j}, j\right)$ may be less than $\max _{j \in J} d\left(i_{j}^{*}, j\right)$. Fortunately, this potential problem can be resolved if we simultaneously solve a series of variant versions of the maximum-density segment problem.

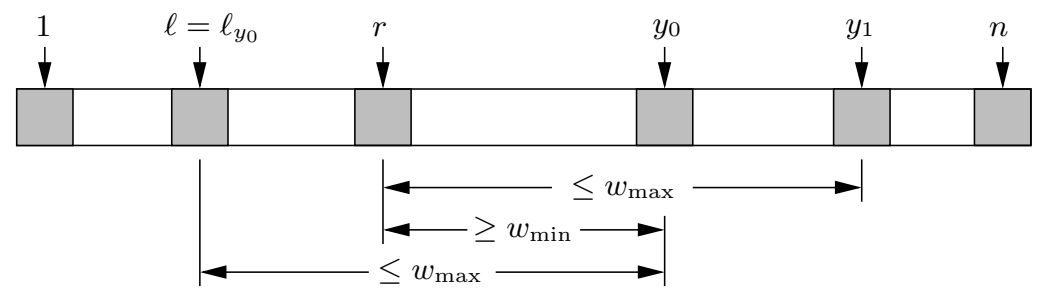

FIG. 4.1. An illustration for the relation among $\ell, r, y_{0}, y_{1}$.

4.1. A variant version of the maximum-density segment problem. Suppose that we are given two indices $r$ and $y_{0}$ with $w\left(r, y_{0}\right) \geq w_{\min }$. Let $X=[\ell, r]$ and $Y=\left[y_{0}, y_{1}\right]$ be two intervals such that $\ell=\ell_{y_{0}}$ and $y_{1}$ is the largest index in $J$ with $w\left(r, y_{1}\right) \leq w_{\max }$. See Figure 4.1 for an illustration. The variant version of the maximum-density segment problem is to look for a maximum-density segment over 
$\operatorname{AlgORIthm~\operatorname {Vmain}}\left(r, y_{0}\right)$.

let $\ell$ be the smallest index in $[1, n]$ with $w\left(\ell, y_{0}\right) \leq w_{\max }$;

let $y_{1}$ be the largest index in $[1, n]$ with $w\left(r, y_{1}\right) \leq w_{\max }$;

let $x_{y_{0}-1}=\ell$;

for $y=y_{0}$ to $y_{1}$ do \{

let $x_{y}=\operatorname{BEST}\left(\max \left(x_{y-1}, \ell_{y}\right), r, y\right)$;

\}

output $\left(x_{y}, y\right)$

FIG. 4.2. Our algorithm for the variant version of the maximum-density segment problem, where function BEST is as defined in Figure 2.2.

all feasible segments $S(x, y)$ with $x \in X, y \in Y$, and $w_{\min } \leq w(x, y) \leq w_{\max }$ such that $d(x, y)$ is maximized.

For each $y \in Y$, let $x_{y}^{*}$ be the largest index $x \in X$ with $w_{\min } \leq w(x, y) \leq w_{\max }$ that maximizes $d(x, y)$. Let $y^{*}$ be an index in $Y$ with $d\left(x_{y^{*}}^{*}, y^{*}\right)=\max _{y \in Y} d\left(x_{y}^{*}, y\right)$. Although solving the variant version can naturally be reduced to computing the index $x_{y}^{*}$ for each index $y \in Y$, the required running time is more than what we can afford. Instead, we compute an index $x_{y} \in X$ with $w_{\min } \leq w\left(x_{y}, y\right) \leq w_{\max }$ for each index $y \in Y$ such that $x_{y^{*}}=x_{y^{*}}^{*}$. By $w\left(r, y_{0}\right) \geq w_{\min }$ and $w\left(r, y_{1}\right) \leq w_{\max }$, one can easily see that, for each $y \in Y, r$ is always the largest index $x \in X$ with $w_{\min } \leq w(x, y) \leq w_{\max }$. Our algorithm for solving the variant problem is as shown in Figure 4.2, presented in a way to emphasize the analogy between VMAIN and MAIN. For example, the index $x_{y}$ in VMAIN is the counterpart of the index $i_{j}$ in MAIN. Also, the index $r$ in VMAIN plays the role of the index $r_{j}$ in MAIN. We have the following lemma whose proof is very similar to that of Theorem 2.2.

LEMMA 4.1. Algorithm VMAIN solves the variant version of the maximum-density problem correctly.

Proof. We prove the theorem by showing $x_{y^{*}}=x_{y^{*}}^{*}$. By $\ell_{y_{0}}=x_{y_{0}-1}=\ell$ and Lemma 2.1, the equality holds if $y^{*}=y_{0}$. The rest of the proof assumes $y^{*}>y_{0}$. By Lemma 2.1 and $\ell_{y^{*}} \leq x_{y^{*}}^{*}$, it suffices to ensure $x_{y^{*}-1} \leq x_{y^{*}}^{*}$. Assume for contradiction that there is an index $y \in\left[y_{0}, y^{*}-1\right]$ with $x_{y-1} \leq x_{y^{*}}^{*}<x_{y}$. By $y<y^{*}$, we know $\ell_{y} \leq x_{y^{*}}^{*}$. By Lemma 2.1 and $\max \left(\ell_{y}, x_{y-1}\right) \leq x_{y^{*}}^{*}<x_{y} \leq r$, we have $d\left(x_{y}, y\right) \geq$ $d\left(x_{y^{*}}^{*}, y\right)$. It follows from (1.1) and $x_{y^{*}}^{*}<x_{y}$ that $d\left(x_{y^{*}}^{*}, y\right) \geq d\left(x_{y^{*}}^{*}, x_{y}-1\right)$. By $\ell_{y^{*}} \leq x_{y^{*}}^{*}<x_{y} \leq r$ and the definition of $y^{*}$, we know $d\left(x_{y^{*}}^{*}, y^{*}\right)>d\left(x_{y}, y^{*}\right)$. It follows from $x_{y^{*}}^{*}<x_{y}$ and (1.1) that $d\left(x_{y^{*}}^{*}, x_{y}-1\right)>d\left(x_{y^{*}}^{*}, y^{*}\right)$. Therefore, $d\left(x_{y}, y\right) \geq$ $d\left(x_{y^{*}}^{*}, y\right) \geq d\left(x_{y^{*}}^{*}, x_{y}-1\right)>d\left(x_{y^{*}}^{*}, y^{*}\right)$, contradicting the definition of $y^{*}$.

Again, the challenge lies in supporting each query to $\phi(i, r-1)$ of BEST in $O(1)$ time during the execution of VMAIN. Fortunately, unlike during the execution of MAIN, where both parameters of $\phi(i, r-1)$ may change, the second parameter $r-1$ is now fixed. Therefore, to support each query to $\phi(i, r-1)$ in $O(1)$ time, we can actually afford $O(r-\ell+1)$ time to compute a data structure $\Psi$ such that $\Psi[i]=\phi(i, r-1)$ for each $i \in[\ell, r-1]$. As a result, the function BEST can be implemented as the function VBEST shown in Figure 4.3. The following lemma ensures the correctness and efficiency of our implementation VARIANT shown in Figure 4.3.

LEMMA 4.2. The implementation VARIANT correctly solves the variant version of the maximum-density segment problem in $O\left(r-\ell+y_{1}-y_{0}+1\right)$ time.

Proof. One can easily verify that if $\Psi[i]=\phi(i, r-1)$ holds for each index $i \in$ 
Algorithm VARIANT $\left(r, y_{0}\right)$.

let $\ell$ be the smallest index in $[1, n]$ with $w\left(\ell, y_{0}\right) \leq w_{\max }$;

let $y_{1}$ be the largest index in $[1, n]$ with $w\left(r, y_{1}\right) \leq w_{\max }$;

call $\operatorname{INIT}(\ell, r-1)$;

let $x_{y_{0}-1}=\ell$;

for $y=y_{0}$ to $y_{1}$ do \{

let $x_{y}=\operatorname{vBEST}\left(\max \left(x_{y-1}, \ell_{y}\right), r, y\right)$;

\}

output $\left(x_{y}, y\right)$

FUNCTION $\operatorname{vBEST}(\ell, r, y)$.

let $x=\ell$;

while $x<r$ and $d(x, \Psi[x]) \leq d(x, y)$ do

let $x=\Psi[x]+1$;

return $x$;

Subroutine $\operatorname{Init}(\ell, r)$

let $\Psi[r]=r$;

for $s=r-1$ downto $\ell$ do \{

let $t=s$;

while $t<r$ and $d(s, t) \geq d(s, \Psi[t+1])$ do

let $t=\Psi[t+1]$;

let $\Psi[s]=t$;

\}

FIG. 4.3. An efficient implementation for the algorithm VMAIN.

$[\ell, r-1]$, then VBEST is a faithful implementation of BEST. Therefore, by Lemma 4.1, the correctness of VARIANT can be ensured by showing that after calling $\operatorname{INIT}(\ell, r-1)$ at step 3 of algorithm VARIANT,$\Psi[i]=\phi(i, r-1)$ holds for each index $i \in[\ell, r-1]$. Note that for brevity of the following proof, we slightly abuse the notation $r$ in Figure 4.3. That is, although the subroutine call at step 3 of algorithm VARIANT is $\operatorname{INIT}(\ell, r-1)$, the second parameter in the definition of subroutine INIT in Figure 4.3 becomes $r$. Let us make it clear that the rest of the proof (i) lets $r$ denote the one in the definition of subroutine $\operatorname{INIT}(\ell, r)$, and (ii) proves that $\Psi[i]=\phi(i, r)$ holds for each index $i \in[\ell, r]$ at the end of the subroutine call to subroutine $\operatorname{INIT}(\ell, r)$.

By step 1 of InIT, we have $\Psi[r]=r=\phi(r, r)$. Now suppose that $\Psi[i]=\phi(i, r)$ holds for each index $i \in[x+1, r]$ right before INIT is about to execute the iteration for index $x \in[\ell, r]$. It suffices to show $\Psi[x]=\phi(x, r)$ after the iteration. Let

$$
Z_{x}=\{x, \Psi[x+1], \Psi[\Psi[x+1]+1], \Psi[\Psi[\Psi[x+1]+1]+1], \ldots, r\} .
$$

Let $\left|Z_{x}\right|$ denote the cardinality of $Z_{x}$. We first show $\phi(x, r) \in Z_{x}$ as follows.

Assume for contradiction that $\phi(x, r) \notin Z_{x}$, i.e., there is an index $z \in Z_{x}$ with $z<\phi(x, r)<\Psi[z+1]=\phi(z+1, r)$. By definition of $\phi$ and (1.1), we have $d(z+1, \phi(x, r))>d(z+1, \phi(z+1, r))>$ $d(\phi(x, r)+1, \phi(z+1, r))$ and $d(\phi(x, r)+1, \phi(z+1, r)) \geq d(x, \phi(z+$ $1, r)) \geq d(x, \phi(x, r))$. By $d(z+1, \phi(x, r))>d(x, \phi(x, r))$ and (1.1), we have $d(x, \phi(x, r))>d(x, z)$, contradicting the definition of $\phi(x, r)$.

For any index $z \in Z_{x}$ with $z<\phi(x, r)$, we know $z<r$ and $\phi(z+1, r)=\Psi[z+1] \leq$ 


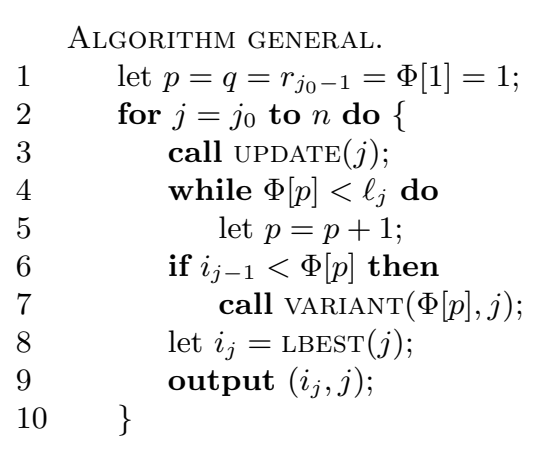

FIG. 4.4. Our algorithm for the general case, where UPDATE and LBEST are defined in Figure 3.2 and VARIANT is defined in Figure 4.3.

$\phi(x, r)$. By $\phi(z+1, r) \leq \phi(x, r) \leq r$ and the definition of $\phi(z+1, r)$, we have $d(z+1, \phi(x, r)) \geq d(z+1, \phi(z+1, r))$. By definition of $\phi(x, r)$ and (1.1), we have $d(x, z) \geq d(x, \phi(x, r)) \geq d(z+1, \phi(x, r))$. By $d(x, z) \geq d(z+1, \phi(z+1, r))$ and (1.1), we have $d(x, z) \geq d(x, \phi(z+1, r))$. Therefore, if $z<\phi(x, r)$, then step 5 of INIT will be executed to increase the value of $z$. Observe that $\phi(x, r)=z<r$ and $\Psi[z+1]>z$ imply $d(x, z)<d(x, \Phi[z+1])$. It follows that as soon as $z=\phi(x, r)$ holds, whether $\phi(x, r)=r$ or not, the value of $\Psi[x]$ will immediately be set to $z$ at step 6 of INIT.

As for the time complexity, we first observe that $\ell$ and $y_{1}$ can be found from $r$ and $y_{0}$ in $O\left(r-\ell+y_{1}-y_{0}+1\right)$ time:

- Let $\ell=r$, and then repeatedly decrease $\ell$ by 1 as long as $w\left(\ell-1, y_{0}\right) \leq w_{\max }$ holds.

- Let $y_{1}=y_{0}$, and then repeatedly increase $y_{1}$ by 1 as long as $w\left(r, y_{1}+1\right) \leq$ $w_{\max }$ holds.

Secondly, one can see that the rest of the implementation also runs in $O\left(r-\ell+y_{1}-\right.$ $\left.y_{0}+1\right)$ time by verifying that throughout the execution of the implementation (a) the while-loop of VBEST runs for $O\left(r-\ell+y_{1}-y_{0}+1\right)$ iterations, and (b) the while-loop of INIT runs for $O(r-\ell+1)$ iterations. To see statement (a), just observe that the value of index $x$ (i) never decreases, (ii) stays in $[\ell, r]$, and (iii) increases by at least one each time step 3 of VBEST is executed. As for statement (b), consider the iteration with index $s$ of the for-loop of INIT. Note that if step 6 of INIT executes $t_{s}$ times in this iteration, then $\left|Z_{s}\right|=\left|Z_{s+1}\right|-t_{s}+1$. Since $\left|Z_{s}\right| \geq 1$ holds for each $s \in X$, we have $\sum_{s \in X} t_{s}=O(r-\ell+1)$, and thus statement (b) holds.

4.2. Our algorithm for the general case. With the help of VARIANT, we have a linear-time algorithm for solving the original maximum-density segment problem as shown in Figure 4.4. Algorithm GENERAL is obtained by inserting four lines of code (i.e., steps $4-7$ of GENERAL) between steps 3 and 4 of LMAIN in order to handle the case $i_{j-1}<\ell_{j}$. Specifically, when $i_{j-1}<\ell_{j}$, we cannot afford to appropriately update the data structure $\Phi$. Therefore, instead of moving $i$ to $\ell_{j}$, steps 4 and 5 move $i$ to $\Phi[p]$, where $p$ is the smallest index with $\ell_{j} \leq \Phi[p]$. Of course, these two steps may cause our algorithm to overlook the possibility of $i_{j} \in\left[i_{j-1}, \Phi[p]-1\right]$, as illustrated in Figure 4.5. This is when the variant version comes in: As shown in the next theorem, we can remedy the problem by calling $\operatorname{VARIANT}(\Phi[p], j)$. 


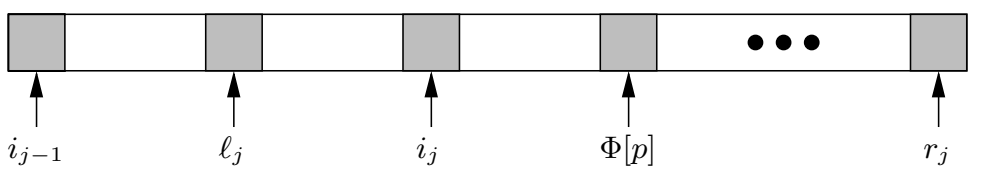

FIG. 4.5. An illustration for the situation when Steps 6 and 7 of GENERAL are needed.

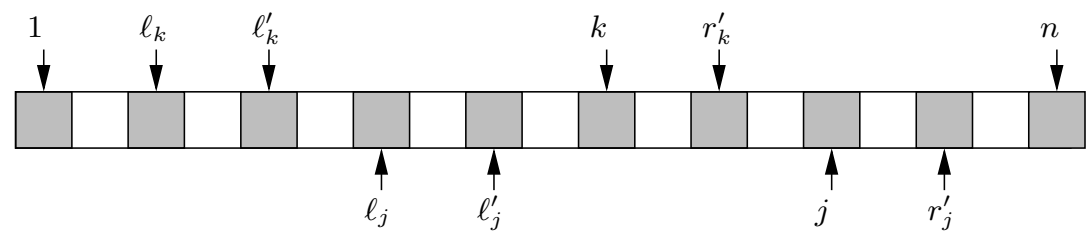

FIG. 4.6. An illustration showing that the overall running time of all subroutine calls to $\operatorname{VARIANT}\left(\ell_{j}^{\prime}, j\right)$ in GENERAL is $O(n)$.

THEOREM 4.3. Algorithm GENERAL solves the maximum-density segment problem in an online manner in linear time.

Proof. We prove the correctness of GENERAL by showing that $i_{j^{*}}^{*} \neq i_{j^{*}}$ implies $i_{j^{*}}^{*}=x_{j^{*}}$. By Lemma 3.1(1), Condition $C_{j}$ holds after the subroutine call UPdATE $(j)$ at step 3 of GENERAL. Observe that steps 4 and 5 of GENERAL, which may increase the value of $p$, do not affect the validity of Condition $C_{j}$. Also, steps 6 and 7 do not modify $p, q$, and $\Phi$. Let $\ell_{j}^{\prime}$ be the value of $\Phi[p]$ right before executing step 8 of GENERAL. By Lemma 3.1(2), the index $i_{j}$ returned by LBEST $(j)$ is the largest index in $\left[\ell_{j}^{\prime}, r_{j}\right]$ that maximizes $d\left(i_{j}, j\right)$. Clearly, $i_{j^{*}}=i_{j^{*}}^{*}$ implies the correctness of GENERAL. If $i_{j^{*}} \neq i_{j^{*}}^{*}$, there must be an index $j \in\left[j_{0}, j^{*}\right]$ with $i_{j-1} \leq i_{j^{*}}^{*}<i_{j}$. It can be proved as follows that $i_{j^{*}}^{*} \leq \ell_{j}^{\prime}-1$.

Assume $\ell_{j}^{\prime} \leq i_{j^{*}}^{*}$ for contradiction. It follows from Lemma 3.1(2) and (1.1) that $d\left(i_{j}, j\right) \geq d\left(i_{j^{*}}^{*}, j\right) \geq d\left(i_{j^{*}}^{*}, i_{j}-1\right)$. By the definition of $j^{*}$, we have $d\left(i_{j^{*}}^{*}, j^{*}\right)>d\left(i_{j}, j^{*}\right)$, which by (1.1) implies $d\left(i_{j^{*}}^{*}, i_{j}-1\right)>$ $d\left(i_{j^{*}}^{*}, j^{*}\right)$. Therefore, $d\left(i_{j}, j\right)>d\left(i_{j^{*}}^{*}, j^{*}\right)$, contradicting the definition of $j^{*}$.

Since $i_{j-1} \leq i_{j^{*}}^{*} \leq \ell_{j}^{\prime}-1$, we know $w\left(\ell_{j}^{\prime}-1, j^{*}\right) \leq w\left(i_{j^{*}}^{*}, j^{*}\right) \leq w_{\max }$. Thus, $S\left(i_{j^{*}}^{*}, j^{*}\right)$ is a feasible segment in the variant version of the maximum-density segment problem for $S$ with respect to indices $r=\ell_{j}^{\prime}$ and $y_{0}=j$. By Lemma 4.2, the subroutine call $\operatorname{VARIANT}\left(\ell_{j}^{\prime}, j\right)$ at step 7 of algorithm GENERAL has to output an index pair $(x, y)$ with $w_{\min } \leq w(x, y) \leq w_{\max }$ and $d(x, y)=d\left(i_{j^{*}}^{*}, j^{*}\right)$.

As for the running time, observe that $q-p \geq 0$ holds throughout the execution of GENERAL. Step 4 of UPDATE, which is the only place that increases the value of $q-p$, increases the value of $q-p$ by 1 for $O(n)$ times. Note that each iteration of the while-loops of GENERAL, LBEST, and UPDATE decreases the value of $q-p$ by 1 . Therefore, to show that the overall running time of GENERAL is $O(n)$, it remains to ensure that all those subroutine calls to VARIANT at step 7 of GENERAL take overall $O(n)$ time. Suppose that $j$ and $k$ are two arbitrary indices with $k<j$ such that GENERAL makes subroutine calls to $\operatorname{VARIANT}\left(\ell_{k}^{\prime}, k\right)$ and $\operatorname{varianT}\left(\ell_{j}^{\prime}, j\right)$. Let $r_{k}^{\prime}$ be the largest index in $[1, n]$ with $w\left(\ell_{k}^{\prime}, r_{k}^{\prime}\right) \leq w_{\max }$. By Lemma 4.2, it suffices to show $\ell_{k}^{\prime}<\ell_{j}$ and $r_{k}^{\prime}<j$ as follows. (See Figure 4.6.) By the definition of GENERAL, we know that $i_{j-1}<\ell_{j}$, which is ensured by the situation illustrated in Figure 4.5. By $k<j$, we have $\ell_{k}^{\prime} \leq i_{j-1}$, implying $\ell_{k}^{\prime}<\ell_{j}$. Moreover, by the definitions of $\ell_{j}$ and 
$r_{k}^{\prime}$, one can easily verify that $\ell_{k}^{\prime}<\ell_{j}$ implies $r_{k}^{\prime}<j$.

It is not difficult to see that our algorithm shown in Figure 4.4 is already capable of processing the input sequence in an online manner, since the only preprocessing required is to obtain $\ell_{j}, r_{j}$, and the prefix sums of $a_{1}, a_{2}, \ldots, a_{j}$ and $w_{1}, w_{2}, \ldots, w_{j}$ (for the purpose of evaluating the density of any segment in $O(1)$ time), which can easily be computed on the fly.

5. Exploiting sparsity for the uniform case. In this section, we assume that the input sequence $S=\left(a_{1}, w_{1}\right),\left(a_{2}, w_{2}\right), \ldots,\left(a_{n}, w_{n}\right)$ is run-length encoded [15], i.e., represented by $m$ pairs $\left(a_{1}^{\prime}, n_{1}\right),\left(a_{2}^{\prime}, n_{2}\right), \ldots,\left(a_{m}^{\prime}, n_{m}\right)$ with $0=n_{0}<n_{1}<n_{2}<\cdots<$ $n_{m}=n$ to signify that $w_{1}=w_{2}=\cdots=w_{n}=1$ and that $a_{i}=a_{j}^{\prime}$ holds for all indices $i$ and $j$ with $n_{j-1}<i \leq n_{j}$ and $1 \leq j \leq m$. Our algorithm for solving the maximum-density problem for the $O(m)$-space representable sequence $S$ is shown in Figure 5.1.

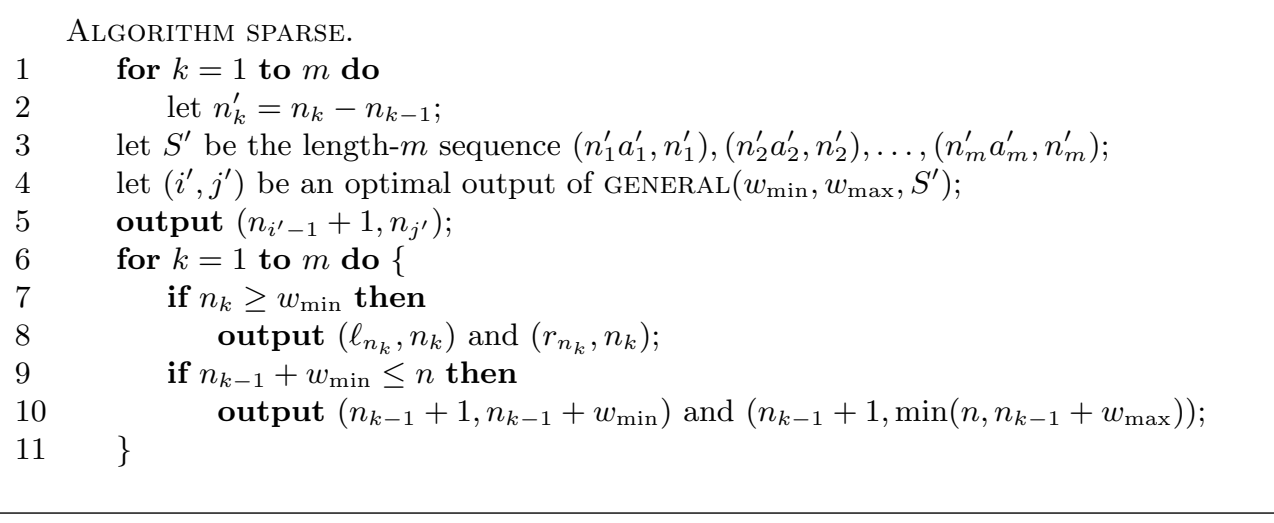

FIG. 5.1. Our algorithm that handles sparse input sequence for the uniform case, where GENERAL is defined in Figure 4.4 .

THEOREM 5.1. Algorithm SPARSE solves the maximum-density problem for the above $O(m)$-space representable sequence in $O(m)$ time.

Proof. By Theorem 4.3, SPARSE runs in $O(m)$ time. Let $S\left(i^{*}, j^{*}\right)$ be a feasible segment with maximum density. We first show that without loss of generality $i^{*}-1 \in$ $\left\{n_{0}, n_{1}, \ldots, n_{m-1}\right\}$ or $j^{*} \in\left\{n_{1}, n_{2}, \ldots, n_{m}\right\}$ holds. More specifically, we show as follows that if $i^{*}-1 \notin\left\{n_{0}, n_{1}, \ldots, n_{m-1}\right\}$ and $j^{*} \notin\left\{n_{1}, n_{2}, \ldots, n_{m}\right\}$, then $S\left(i^{*}+\right.$ $\left.1, j^{*}+1\right)$ is also a feasible segment with maximum density.

By $i^{*}-1 \notin\left\{n_{0}, n_{1}, \ldots, n_{m-1}\right\}$, we know $a_{i^{*}-1}=a_{i^{*}}$. By $j^{*} \notin$ $\left\{n_{1}, n_{2}, \ldots, n_{m}\right\}$, we know $a_{j^{*}}=a_{j^{*}+1}$. It follows from the optimality of $S\left(i^{*}, j^{*}\right)$ that $a_{i^{*}} \geq a_{j^{*}+1}$ and $a_{i^{*}-1} \leq a_{j^{*}}$, implying $a_{i^{*}-1}=a_{i^{*}}=$ $a_{j^{*}}=a_{j^{*}+1}$. Therefore, $S\left(i^{*}+1, j^{*}+1\right)$ is also a maximum-density segment.

It remains to show that our algorithm works correctly for each possible case.

- Case 1: $i^{*}-1 \in\left\{n_{0}, n_{1}, \ldots, n_{m-1}\right\}$ and $j^{*} \in\left\{n_{1}, n_{2}, \ldots, n_{m}\right\}$. Clearly, steps $1-5$ of SPARSE take care of this case.

- Case 2: $i^{*}-1 \notin\left\{n_{0}, n_{1}, \ldots, n_{m-1}\right\}$ and $j^{*} \in\left\{n_{1}, n_{2}, \ldots, n_{m}\right\}$. Clearly, if $i^{*} \in\left\{\ell_{j^{*}}, r_{j^{*}}\right\}, S\left(i^{*}, j^{*}\right)$ can be discovered by steps 7 and 8 of SPARSE. Since $i^{*}-1 \notin\left\{n_{0}, n_{1}, \ldots, n_{m-1}\right\}$, we have $a_{i^{*}-1}=a_{i^{*}}$. If $a_{i^{*}-1}=a_{i^{*}} \neq d\left(i^{*}, j^{*}\right)$, then by (1.1) we have either $d\left(i^{*}-1, j^{*}\right)>d\left(i^{*}, j^{*}\right)$ or $d\left(i^{*}+1, j^{*}\right)>d\left(i^{*}, j^{*}\right)$, 
which implies that either $S\left(i^{*}-1, j^{*}\right)$ or $S\left(i^{*}+1, j^{*}\right)$ is infeasible, and thus $i^{*} \in\left\{\ell_{j^{*}}, r_{j^{*}}\right\}$. On the other hand, if $a_{i^{*}-1}=a_{i^{*}}=d\left(i^{*}, j^{*}\right)$ and $i^{*} \neq \ell_{j}^{*}$, then $S\left(i^{*}-1, j^{*}\right)$ is also a feasible segment with maximum density. We can continue the same argument until we have a maximum-density segment $S\left(i, j^{*}\right)$ such that either $i-1 \in\left\{n_{0}, n_{1}, \ldots, n_{m-1}\right\}$, which is handled in Case 1 , or $i=\ell_{j^{*}}$, which is handled by steps 7 and 8 of SPARSE.

- Case 3: $i^{*}-1 \in\left\{n_{0}, n_{1}, \ldots, n_{m-1}\right\}$ and $j^{*} \notin\left\{n_{1}, n_{2}, \ldots, n_{m}\right\}$. The proof of this case, omitted for brevity, is very similar to that of Case 2 .

The theorem is proved.

Acknowledgments. We thank Yi-Hsuan Hsin and Hsu-Cheng Tsai for discussions during the preliminary stage of this research. We thank Tien-Ching Lin for comments.

\section{REFERENCES}

[1] N. N. Alexandrov and V. V. Solovyev, Statistical significance of ungapped sequence alignments, in Proceedings of the Pacific Symposium on Biocomputing, Maui, HI, 1998, World Scientific, River Edge, NJ, Vol. 3, pp. 461-470.

[2] G. BARHARDI, Isochores and the evolutionary genomics of vertebrates, Gene, 241 (2000), pp. 317.

[3] G. Bernardi and G. Bernardi, Compositional constraints and genome evolution, J. Molec. Evolution, 24 (1986), pp. 1-11.

[4] H. Brönnimann and B. Chazelle, Optimal slope selection via cuttings, Comput. Geom., 10 (1998), pp. 23-29.

[5] B. Charlesworth, Genetic recombination: Patterns in the genome, Current Biol., 4 (1994), pp. $182-184$.

[6] R. Cole, J. S. Salowe, W. L. Steiger, and E. Szemerédi, An optimal-time algorithm for slope selection, SIAM J. Comput., 18 (1989), pp. 792-810.

[7] L. Duret, D. Mouchiroud, And C. Gautier, Statistical analysis of vertebrate sequences reveals that long genes are scarce in GC-rich isochores, J. Mol. Evol., 40 (1995), pp. 308371.

[8] A. Eyre-WALKer, Evidence that both $G+C$ rich and $G+C$ poor isochores are replicated early and late in the cell cycle, Nucleic Acids Res., 20 (1992), pp. 1497-1501.

[9] A. Eyre-Walker, Recombination and mammalian genome evolution, Proc. Roy. Soc. London Ser. B, 252 (1993), pp. 237-243.

[10] J. FILIPSKI, Correlation between molecular clock ticking, codon usage fidelity of DNA repair, chromosome banding and chromatin compactness in germline cells, FEBS Lett., 217 (1987), pp. 184-186.

[11] M. P. Francino And H. Ochman, Isochores result from mutation not selection, Nature, 400 (1999), pp. 30-31.

[12] S. M. Fullerton, A. B. Carvalho, and A. G. Clark, Local rates of recombination are positively correlated with GC content in the human genome, Mol. Biol. Evol., 18 (2001), pp. 1139-1142.

[13] M. H. Goldwasser, M.-Y. KaO, And H.-I. Lu, Fast algorithms for finding maximum-density segments of a sequence with applications to bioinformatics, in Proceedings of the Second International Workshop on Algorithms in Bioinformatics (Rome, Italy, 2002), R. Guigó and D. Gusfield, eds., Lecture Notes in Comput. Sci. 2452, Springer-Verlag, New York, 2002, pp. 157-171.

[14] M. H. Goldwasser, M.-Y. KAO, AND H.-I. Lu, Linear-time algorithms for computing maximum-density sequence segments with bioinformatics applications, J. Comput. System Sci., to appear.

[15] S. W. GolOmB, Run-length encodings, IEEE Trans. Inform. Theory, 12 (1966), pp. 399-401.

[16] P. Guldberg, K. Gronbak, A. Aggerholm, A. Platz, P. thor Straten, V. Ahrenkiel, P. Hokland, And J. Zeuthen, Detection of mutations in GC-rich DNA by bisulphite denaturing gradient gel electrophoresis, Nucleic Acids Res., 26 (1998), pp. 1548-1549.

[17] W. Henke, K. Herdel, K. Jung, D. Schnorr, and S. A. Loening, Betaine improves the PCR amplification of GC-rich DNA sequences, Nucleic Acids Res., 25 (1997), pp. 3957-3958.

[18] G. P. Holmquist, Chromosome bands, their chromatin flavors, and their functional features, Am. J. Hum. Genet., 51 (1992), pp. 17-37. 
[19] X. Huang, An algorithm for identifying regions of a DNA sequence that satisfy a content requirement, Comput. Appl. Biosci., 10 (1994), pp. 219-225.

[20] K. Ikehara, F. Amada, S. Yoshida, Y. Mikata, and A. Tanaka, A possible origin of newly born bacterial genes: Significance of GC-rich nonstop frame on antisense strand, Nucleic Acids Res., 24 (1996), pp. 4249-4255.

[21] R. B. Inman, A denaturation map of the 1 phage DNA molecule determined by electron microscopy, J. Mol. Biol., 18 (1966), pp. 464-476.

[22] I. P. Ioshikhes and M. Q. Zhang, Large-scale human promoter mapping using CpG islands, Nature Genet., 26 (2000), pp. 61-63.

[23] R. Jin, M.-E. Fernandez-Beros, And R. P. Novick, Why is the initiation nick site of an AT-rich rolling circle plasmid at the tip of a GC-rich cruciform?, EMBO J., 16 (1997), pp. $4456-4466$.

[24] M. J. KATZ And M. ShariR, Optimal slope selection via expanders, Inform. Process. Lett., 47 (1993), pp. 115-122.

[25] S. K. KIM, Linear-time algorithm for finding a maximum-density segment of a sequence, Inform. Process. Lett., 86 (2003), pp. 339-342.

[26] Y.-L. Lin, X. HuAnG, T. JiAnG, AND K.-M. ChAO, MAVG: Locating nonoverlapping maximum average segments in a given sequence, Bioinformatics, 19 (2003), pp. 151-152.

[27] Y.-L. Lin, T. JiAng, AND K.-M. ChaO, Algorithms for locating the length-constrained heaviest segments, with applications to biomolecular sequence analysis, J. Comput. System Sci., 65 (2002), pp. 570-586.

[28] G. Macaya, J.-P. Thiery, And G. Bernardi, An approach to the organization of eukaryotic genomes at a macromolecular level, J. Mol. Biol., 108 (1976), pp. 237-254.

[29] C. S. Madsen, C. P. Regan, And G. K. Owens, Interaction of CArG elements and a GC-rich repressor element in transcriptional regulation of the smooth muscle myosin heavy chain gene in vascular smooth muscle cells, J. Biol. Chem., 272 (1997), pp. 29842-29851.

[30] J. MatoušEk, Randomized optimal algorithm for slope selection, Inform. Process. Lett., 39 (1991), pp. 183-187.

[31] S.-I. Murata, P. Herman, and J. R. Lakowicz, Texture analysis of fluorescence lifetime images of AT- and GC-rich regions in nuclei, J. Histochem. Cytochem., 49 (2001), pp. 14431452.

[32] A. Nekrutenko and W.-H. Li, Assessment of compositional heterogeneity within and between eukaryotic genomes, Genome Res., 10 (2000), pp. 1986-1995.

[33] U. Ohler, H. Niemann, G. Liao, and G. M. Rubin, Joint modeling of DNA sequence and physical properties to improve eukaryotic promoter recognition, Bioinformatics, 17 (2001), pp. S199-S206.

[34] P. Rice, I. Longden, And A. Bleasby, EMBOSS: The European molecular biology open software suite, Trends Genet., 16 (2000), pp. 276-277.

[35] L. ScOtto And R. K. Assoian, A GC-rich domain with bifunctional effects on $m R N A$ and protein levels: Implications for control of transforming growth factor beta 1 expression, Mol. Cell. Biol., 13 (1993), pp. 3588-3597.

[36] P. H. Sellers, Pattern recognition in genetic sequences by mismatch density, Bull. Math. Biol., 46 (1984), pp. 501-514.

[37] P. M. Sharp, M. Averof, A. T. Lloyd, G. Matassi, and J. F. Peden, DNA sequence evolution: The sounds of silence, Philos. Trans. Soc. Lond. B Biol. Sci., 349 (1995), pp. 241247.

[38] P. Soriano, M. Meunier-Rotival, And G. Bernardi, The distribution of interspersed repeats is nonuniform and conserved in the mouse and human genomes, Proc. Natl. Acad. Sci. USA, 80 (1983), pp. 1816-1820.

[39] N. Stojanovic, L. Florea, C. Riemer, D. Gumucio, J. Slightom, M. Goodman, W. Miller, AND R. Hardison, Comparison of five methods for finding conserved sequences in multiple alignments of gene regulatory regions, Nucleic Acids Res., 27 (1999), pp. 3899-3910.

[40] N. Sueoka, Directional mutation pressure and neutral molecular evolution, Proc. Natl. Acad. Sci. USA, 80 (1988), pp. 1816-1820.

[41] Z. Wang, E. Lazarov, M. O'Donnel, and M. F. Goodman, Resolving a fidelity paradox: Why Escherichia coli DNA polymerase II makes more base substitution errors in At- compared to GC-rich DNA, J. Biol. Chem., 277 (2002), pp. 4446-4454.

[42] K. H. Wolfe, P. M. Sharp, And W.-H. Li, Mutation rates differ among regions of the mammalian genome, Nature, 337 (1989), pp. 283-285.

[43] Y. Wu, R. P. Stulp, P. Elfferich, J. Osinga, C. H. Buys, and R. M. Hofstra, Improved mutation detection in GC-rich DNA fragments by combined DGGE and CDGE, Nucleic 
Acids Res., 27 (1999), article e9.

[44] S. Zoubak, O. Clay, AND G. Bernardi, The gene distribution of the human genome, Gene, 174 (1996), pp. 95-102. 